\title{
Le télétravail, un objet sans désir ?
}

\author{
Anne AGUILERA, \\ Université Paris Est, IFSTTAR, Laboratoire Ville Mobilité Transport (LVMT) \\ anne.aguilera@ifsttar.fr \\ Virginie LETHIAIS, \\ Telecom Bretagne, Marsouin \\ virginie.lethiais@telecom-bretagne.eu \\ Alain RALLET, \\ RITM, Université Paris-Sud \\ alain.rallet@u-psud.fr \\ Laurent PROULHAC \\ Université Paris Est, IFSTTAR, Laboratoire Ville Mobilité Transport (LVMT) \\ laurent.proulhac@enpc.fr
}

\section{Résumé}

L'objectif de cet article est d'expliquer les écarts entre les fortes attentes sociétales attachées au télétravail et la faiblesse de sa pratique en France. Sur la base de trois enquêtes, nous montrons que le télétravail est non seulement un phénomène limité, mais qu'il est principalement lié à des arrangements informels, et enfin qu'il n'est plébiscité ni par les salariés ni par les entreprises. Les principales raisons avancées concernent ses avantages incertains et ses coûts immédiats. La conclusion discute les éléments de contexte qui pourraient modifier les choix des différents agents.

\section{Mots-clés}

Télétravail, barrières, attentes sociétales, TIC, organisation du travail, France.

\begin{abstract}
The aim of this article is to explain the gap between high social expectations and the reality of home-based telework using three French databases which give information about employers but also employees. We highlight that telework is not only a fairly restricted phenomenon but also one that lacks impetus and is mainly an informal working arrangement. The main reasons raised by both employees and employers are the uncertain advantages coupled with
\end{abstract}


immediate disadvantages. The conclusion examines different contextual factors that could alter this cost-benefits dilemma and foster the development of telework.

\section{Keywords}

Telework, informal telework, barriers, social expectations, ICT, work organization, France. 


\section{Introduction}

Le télétravail, c'est à dire d'un travail réalisé hors du lieu de travail habituel mais connecté à celui-ci par des moyens de télécommunication, est une éternelle promesse : son avenir semble toujours devant lui. Depuis les années 70, les déclarations se sont succédé pour prédire son développement imminent. Ainsi, en 1971, AT\&T affirmait qu'en 1990, tous les américains seraient des télétravailleurs (Huws, 1984). En France, des prédictions plus prudentes mais néanmoins optimistes sont régulièrement affichées. Dans un rapport du Centre d'Analyse Stratégique commandé en novembre 2009 on peut lire que «le télétravail a un fort potentiel de développement qui pourrait concerner jusqu'à 50\% de la population active en 2015, contre $30 \%$ aujourd'hui $»{ }^{1}$. Plus récemment, un rapport de la Chambre de Commerce et de l'Industrie de Paris affirme qu' «Il est consensuellement admis que le télétravail recèle un important potentiel de croissance économique, socialement et écologiquement responsable. Non seulement les postes éligibles au télétravail sont nombreux mais, de surcroît, le télétravail est plébiscité tant par les salariés que par les employeurs. »².

En réalité le télétravail est loin d'avoir tenu ces promesses. Inégal selon les pays, son développement reste marginal, en tous cas loin des prédictions. Même s'il est impossible d'obtenir une mesure fiable et comparable du télétravail, sa définition variant d'une enquête à l'autre, les chiffres annoncés par le cabinet Greenworking ${ }^{3}$ sont loin des attentes : 12,4\% de salariés français télétravaillent au moins huit heures par mois en 2012.

Sa quasi-stagnation ne l'empêche pas de faire l'objet de campagnes de relance périodiques qui lui promettent un prochain décollage. Le principal argument tient à la force des demandes sociales. Le télétravail est supposé accroître le bien-être des salariés : baisse de la fatigue due aux déplacements pendulaires, contexte de travail moins stressant, meilleur équilibre entre vie professionnelle et vie privée. Il est aussi supposé apporter d'importants gains économiques aux entreprises : baisse des coûts immobiliers et plus grande productivité du travail. Les autorités publiques en attendent enfin une baisse des coûts sociaux associés aux déplacements domicile-travail: diminution des coûts de transport, de pollution et de congestion. L'enrichissement des moyens de communication à distance et l'augmentation des contraintes environnementales renforcent ces attentes.

\footnotetext{
${ }^{1}$ Centre d'analyse stratégique (2009), «Le développement du télétravail dans la société numérique de demain », rapport remis au premier ministre, novembre.

${ }^{2}$ Denis D. (2011), Dynamiser le télétravail : un enjeu décisif pour la croissance et l'emploi, Rapport de la Chambre de Commerce et d'Industrie de Paris.

3 «Le télétravail dans les grandes entreprises françaises: Comment la distance transforme nos modes de travail », Synthèse remise au Ministère chargé de l'industrie, de l'énergie et de l'économie numérique, mai 2012.
} 
L'objectif de cet article est d'expliquer le décalage entre les attentes sociales et la réalité du télétravail. La $1^{\text {ère }}$ section précise notre définition du télétravail. La $2^{\text {ème }}$ section propose un état des lieux du télétravail en France à partir de trois bases de données récentes. La $3^{\text {ème }}$ section établit que le télétravail est non seulement un phénomène peu étendu mais qu'il est également sans dynamique réelle. La section 4 en examine les raisons, tant du point de vue des salariés que de celui des entreprises. La conclusion discute les éléments de contexte qui pourraient modifier les comportements des différents agents.

\section{Définition du télétravail et données utilisées}

Il existe une grande confusion autour de la notion de télétravail et de sa mesure, car elle recouvre des phénomènes différents. La littérature propose d'ailleurs de nombreuses typologies (Devillers, 2003 ; Morel-A-Lhuissier, 2007 ; Taskin et Vendramin, 2004). Et la tentation est parfois forte de mélanger certaines de ces variétés sous l'effet faussement unificateur des moyens de télécommunications, ou bien d'inclure des individus dont l'activité a, par nature, toujours été réalisée majoritairement hors des locaux de leur entreprise. Ainsi, une récente étude sur la diffusion du télétravail dans les PME italiennes inclut abusivement les salariés travaillant dans les locaux des clients de leur entreprise dans les effectifs des télétravailleurs... (Neirotti et al., 2013).

\subsection{Une définition en cinq critères}

Notre définition du télétravail est basée sur cinq critères : la nature de la relation salariale, la contrainte spatiale du travail (pourrait-il ou non être réalisé dans le cadre du lieu principal de travail ?), l'échelle géographique (locale, nationale, internationale), la nature des lieux (domicile, télécentres...) et enfin la fréquence (occasionnelle/ régulière, temps passé).

\section{La relation salariale}

Dans cet article, le télétravail concerne un travail reposant sur un contrat de travail salarié : le télétravailleur est un salarié de l'établissement par rapport auquel est défini le travail distant. Sinon, le travail est exercé dans le cadre d'une relation de service entre l'établissement et le prestataire, individuel ou non. On trouve dans cette dernière catégorie aussi bien le pigiste, le consultant ou le designer travaillant chez lui que le sous-traitant informatique en Inde ou une entreprise de «télésecrétariat» dans une zone rurale. Ce sont des téléservices dont le développement pose des problèmes différents du télétravail stricto sensu en matière de coordination, de supervision et d'encadrement légal du travail réalisé à distance. 
La limitation du télétravail au travail salarié conduit à ne considérer que le temps effectué pendant les heures de travail de l'entreprise. Est ainsi exclu le travail effectué le soir à la maison, sauf si le contrat le prend en compte.

\section{La contrainte spatiale}

Nous définissons le télétravail comme un travail qui aurait pu être réalisé dans les locaux de l'employeur mais qui est effectué hors de ces locaux, à domicile ou dans un bureau distant. Cela exclut le travail dont la caractéristique intrinsèque est d'être effectué à l'extérieur des locaux de l'employeur, que ce travail soit exercé de manière mobile (chauffeur routier, chauffeur de taxi, etc.) ou dans un autre lieu, celui d'un client par exemple ${ }^{4}$. En effet, ces salariés appelés nomades sont distants de l'entreprise pour des raisons tenant à la nature de leur travail et non dans le cadre d'une organisation spécifique du travail. Piloté par des moyens de communication, ce type d'activité s'est transformé de manière importante et s'est probablement accru ${ }^{5}$, mais il ne saurait être assimilé à du télétravail.

Par ailleurs, nous ne considérons pas comme étant du télétravail le travail effectué par des salariés dont le lieu effectif de travail est celui de l'entreprise, mais qui sont amenés à travailler dans des lieux variables (gares, salons d'aéroports, bureaux temporaires, moyens de transports, hôtels, cafés...) grâce à la portabilité croissante des TIC. Il est du reste difficile de mesurer cette forme de télétravail, car la question de savoir quel est le temps de travail passé dans des lieux temporaires par un salarié (dont le lieu habituel de travail est celui de l'entreprise) n'est jamais posée dans les enquêtes sur le télétravail ni dans celles sur les pratiques de mobilité, qui s'intéressent aux lieux fixes (domicile, télécentres) autres que le lieu habituel de travail. Outre cette difficulté de mesure, le travail dans des lieux variables ${ }^{6}$ n'a pas été intégré à notre analyse car elle ne soulève pas les mêmes questions que celles que nous souhaitons aborder ici.

\footnotetext{
${ }^{4}$ Beaucoup d'enquêtes sur le télétravail incluent hélas ces salariés. C'est le cas de celle de l'INSEE (INSEE, 2009).

${ }^{5}$ L'augmentation du nombre de salariés nomades n'est pas facile à établir car le lieu effectif de travail est mal renseigné par les enquêtes statistiques (Crague, 2003).

${ }^{6}$ Il nous semble d'ailleurs que l'on devrait s'efforcer de mieux connaître le télétravail mobile des travailleurs fixes. Car, favorisé par les outils portables de communication, il est en augmentation et fait l'objet d'une littérature croissante (Torre, 2008, Rallet et Torre, 2009, Bathelt et Schuldt, 2008) en raison de la valeur ajoutée par ce temps de travail. Sa prise en compte permettrait aussi de dissocier le télétravail de son substrat implicite, lié aux attentes sociales rappelées plus haut : l'économie de déplacements. Si en effet le temps passé en mobilité hors de l'entreprise par des salariés qui y travaillent habituellement n'est plus déconnecté du temps de l'entreprise et crée pour cette raison une valeur nouvelle, alors la positivité du télétravail ne peut plus être représentée par la seule économie de déplacements puisqu'une part de celui-ci s'explique par une valorisation productive de la mobilité.
} 


\section{Dissocier le télétravail de la coopération à distance}

Une conception très extensive du télétravail inclut la coordination à distance intra ou interentreprises de salariés au moyen des TIC. Télétravail est alors confondu avec «travail à distance ». Nous rejetons cette confusion pour deux raisons. Tout d'abord, elle conduirait à ce qu'un grand nombre de salariés soient des télétravailleurs qui s'ignorent, dès lors qu'ils communiquent à distance avec des collègues ou des partenaires. Elle ne répond pas ensuite à la problématique du télétravail qui consiste à se demander si des salariés qui travaillent dans un lieu et s'y coordonnent avec d'autres ne pourraient pas le faire hors de ce lieu.

\section{L'échelle géographique}

Notre définition du télétravail est jusqu'ici indifférente à l'échelle de la distance entre les lieux. Néanmoins le télétravail à l'échelle internationale, appelé souvent travail off shore, rentre dans une autre catégorie, celui du travail à distance visant à exploiter les différentiels de coûts salariaux. Le télétravail à l'échelle nationale (délocaliser le travail de secrétariat dans une zone rurale par exemple) prend souvent la forme d'un travail externalisé, c'est-à-dire une prestation de service. Les débats autour du télétravail concernent aujourd'hui principalement l'échelle locale ou régionale, la préoccupation centrale étant d'alléger les coûts impliqués par les grandes agglomérations urbaines. Une autre raison milite pour une définition localerégionale du télétravail : la nécessité du maintien pour les salariés d'un contact hebdomadaire périodique entre le lieu habituel de travail et le lieu de télétravail.

\section{La fréquence}

Le télétravail effectué de manière occasionnelle n'est généralement pas considéré comme du travail. Celui-ci implique une régularité (hebdomadaire), cependant le temps régulier passé à télétravailler varie selon les enquêtes. Ainsi certaines enquêtes considèrent qu'il y a télétravail à partir d'une demi-journée télétravaillée, d'autres à partir de la journée... Il nous semble que la demi-journée est une condition minimale acceptable dès lors qu'elle est régulière. Le plus souvent, le télétravail est une activité à temps partiel, le salarié partageant son temps entre le lieu habituel de travail et le lieu de télétravail.

Nous avons pourtant choisi dans les deux enquêtes régionales que nous avons réalisées, de ne pas définir le télétravail en termes de fréquence afin de recueillir des informations sur l'ensemble des pratiques. Nous disposons ainsi de deux mesures du télétravail : une mesure au sens stricte dans laquelle la fréquence minimale du télétravail est d'une demi-journée par 
semaine ; une mesure plus large, qui comprend un travail réalisé à domicile ou dans un télécentre de manière plus ponctuelle.

Au final, le télétravail considéré dans cet article est un travail salarié qui aurait pu être réalisé dans les locaux de l'employeur mais qui est effectué régulièrement hors de ces locaux, à une échelle locale ou régionale, au lieu du domicile ou dans un bureau distant. Cette définition est très proche de certaines définitions officielles, par exemple celle de l'accord cadre européen sur le télétravail de 2007, repris ensuite par plusieurs accords professionnels nationaux. Elle a l'avantage de ne pas mélanger des formes de travail aux logiques différentes et de se centrer sur les attentes sociales dont le télétravail est l'objet : bien-être des salariés, coûts et efficacité pour les entreprises, et enfin réduction des coûts sociaux du navettage pour les autorités publiques.

\subsection{Trois sources de données}

Pour mesurer et caractériser le télétravail tel qu'il vient d'être défini, notre réflexion s'appuie sur les données de trois enquêtes qui offrent des regards très complémentaires sur la pratique du télétravail en France, l'une à l'échelle nationale et les deux autres dans un territoire spécifique (la Région Bretagne). Il ne s'agit pas de comparer directement ces enquêtes, qui n'ont ni le même échantillon ni exactement la même définition du télétravail, mais bien, en l'absence de données adéquates, de contribuer à expliciter les raisons du décalage entre attentes sociétales et pratiques du télétravail, du point de vue des entreprises mais également des salariés.

La première source de données est une enquête sur la mobilité des Français qui comporte une question sur la pratique du télétravail à domicile. Réalisée en 2008 par l'INSEE auprès d'un échantillon représentatif de la population française, l'Enquête Nationale Transport et Déplacements (ENTD) décrit de façon très complète les déplacements des Français pour le travail, les achats, les loisirs, etc. Les actifs ayant un lieu d'emploi hors de leur domicile sont également interrogés sur leur pratique du télétravail, celui-ci étant défini comme le fait de travailler à domicile au lieu d'aller sur son lieu de travail habituel avec l'accord de l'employeur. On sait aussi si cette pratique est régulière ou occasionnelle, même si la

\footnotetext{
7 « le télétravail est une forme d'organisation et/ou de réalisation du travail, utilisant les technologies de l'information, dans le cadre d'un contrat ou d'une relation d'emploi, dans laquelle un travail, qui aurait également pu être réalisé dans les locaux de l'employeur, est effectué hors de ces locaux de façon régulière »
} 
fréquence n'est pas précisée. Les personnes dont le lieu de travail est le domicile ne sont pas comptabilisées dans la population des télétravailleurs à domicile.

Les deux autres enquêtes ont été réalisées par le GIS MARSOUIN ${ }^{8}$ en 2012, dans le cadre de l'observatoire OPSIS (Observatoire régional des usages du numérique) ${ }^{9}$. Un volet de questions spécifiques sur le travail à distance a été intégré par les auteurs de cet article dans chacune de ces enquêtes. Ces dernières sont circonscrites à la Région Bretagne et concernent pour l'une un échantillon représentatif de 1294 PME et pour l'autre un échantillon représentatif de 2000 résidents. Elles permettent donc de confronter le regard des entreprises et des salariés sur le télétravail, en tant que pratique effective pour certains ou bien potentielle pour d'autres. L'enquête auprès des salariés ne concerne que le télétravail à domicile, tandis que l'enquête auprès des entreprises considère aussi le télétravail depuis un bureau distant.

\section{Le grand décalage entre le discours sur le télétravail et la réalité}

Les trois enquêtes confirment que la pratique du télétravail est très limitée et réservée à certaines professions mais aussi... aux Parisiens !

\subsection{Une pratique limitée}

Les lieux dans lesquels nous travaillons apparaissent plus variés qu'auparavant (Crague, 2003), sans qu'on sache toutefois véritablement ce qui relève d'une multiplication effective des endroits dans lesquels il est possible de travailler, ou surtout d'une diversification des tâches que l'on peut effectivement réaliser en dehors de son lieu de travail habituel. Les TIC sont un des éléments clés de ces évolutions, et participent en particulier à l'enchevêtrement croissant entre vie privée et vie professionnelle (Boboc et Dhaleine, 2008).

Le travail tend à envahir le domicile, mais aussi tout un ensemble de tiers-lieux : télécentres, espaces de co-working, bureaux temporaires... (Aguiléra et al., 2013; Crague, 2003 ; Moriset, 2004). Toutefois ces pratiques sont encore très mal mesurées. Le travail réalisé par A. Boboc et al. (2007) est l'un des rares à avancer des chiffres nationaux, qui montrent que seulement $55 \%$ des actifs français travaillent exclusivement dans un seul lieu : pour $51 \%$ il s'agit d'un établissement, et pour $4 \%$ de leur domicile. Parmi les $45 \%$ d'actifs restants, seuls 9\% sont des nomades qui n'ont pas de lieu de travail fixe et sont toujours en déplacement. Les

\footnotetext{
${ }^{8}$ Môle armoricain de recherche sur la société de l'information et les usages d'Internet (http://www.marsouin.org) ${ }^{9}$ L'observatoire OPSIS réalise régulièrement, à l'échelle de la Bretagne, des enquêtes auprès des PME et des particuliers portant sur les usages du numérique.
} 
autres (36\%) déploient donc leur activité professionnelle entre leur établissement, leur domicile et des tiers-lieux. Malgré tout, le télétravail à domicile semble encore très limité, ce que confirme l'ENTD qui permet d'estimer la part des télétravailleurs réguliers ou occasionnels à domicile à seulement 6,8\% des actifs français en 2008. Si on ne prend en compte que les actifs ayant déclaré un lieu de travail fixe hors de leur domicile, alors cette proportion atteint $8 \%$ : 5\% sont des télétravailleurs occasionnels, et 3\% des télétravailleurs réguliers. Ces chiffres concordent avec ceux de l'enquête menée auprès des actifs bretons : en effet 7\% d'entreu-eux (dont le lieu de travail n'est pas le domicile) déclarent télétravailler au moins une journée complète à leur domicile : c'est une pratique régulière (au moins un jour par semaine) pour $3 \%$, et seulement occasionnelle pour les $4 \%$ restants.

Du côté des entreprises, l'INSEE estimait en 2009 que 22\% des entreprises implantées en France comptaient au moins un salarié travaillant hors des locaux de l'entreprise au moins une demi-journée par semaine tout en ayant accès au système informatique, soit une définition assez large du télétravail incluant les pratiques de nomadisme (INSEE, 2009). L'enquête réalisée en Bretagne montre que $18 \%$ des PME déclarent qu'au moins un de leurs salariés pratique le télétravail à domicile ou dans un bureau distant au sens large (c'est-à-dire quelle que soit la fréquence), ce chiffre tombant à $12,4 \%$ si on se limite au télétravail effectué au moins une demi-journée par semaine. Ces chiffres sont donc bien inférieurs à ceux de l'INSEE, notamment parce que le travail nomade est exclu de notre définition du télétravail, de même que sont exclus les grandes entreprises et Paris alors qu'ils sont plus consommateurs de télétravail. Un autre résultat de l'enquête menée auprès des PME bretonnes et que seule une faible part des salariés est concernée : dans $41 \%$ des entreprises concernées seul un salarié pratique le télétravail au sens strict et dans les trois-quarts des cas, le télétravail concerne moins de 4 salariés.

\subsection{Le télétravail informel comme modèle dominant}

Plusieurs auteurs ont mis en avant le développement du télétravail informel, cette pratique faisant le plus souvent l'objet d'un accord tacite, non formalisé, entre l'employeur et le salarié (Farrah et Dagen, 1993 ; Taskin et Vendramin, 2004). Les deux enquêtes réalisées en Bretagne confirment ce caractère largement informel. Parmi les entreprises qui déclarent pratiquer le travail à distance, seulement $41 \%$ l'ont inscrit au contrat de travail de leurs salariés, tandis que $43 \%$ envisagent de l'inscrire et que près de $11 \%$ ne l'ont pas inscrit et n'ont pas l'intention de le faire. En fait, la pratique du télétravail est d'autant plus officialisée 
qu'elle est fréquente. En effet, les entreprises qui déclarent faire travailler des salariés à distance toute la semaine sont $62 \%$ à l'avoir officialisé. Celles qui déclarent faire travailler des salariés à distance plusieurs jours par semaine sont la moitié à l'avoir inscrit dans le contrat de travail. Mais ce chiffre tombe à $25 \%$ pour les entreprises qui pratiquent le travail à distance au moins une demi-journée par semaine, et à $23 \%$ seulement pour une pratique encore plus occasionnelle.

Finalement, si on ne considère que les entreprises dont un salarié au moins travaille à distance au minimum une demi-journée par semaine et qui ont inscrit cette organisation au contrat de travail, cela ne représente plus que 6,5\% des entreprises de notre échantillon (un peu moins de $8 \%$ si on ajoute celles qui disent avoir prévu de l'officialiser). Pour résumer, on peut dire que le télétravail est assez peu répandu, et qu'il correspond encore surtout à des formes d'arrangement non contractualisées.

\subsection{Une sélectivité spatiale et professionnelle}

Les trois enquêtes convergent également sur le fait que le télétravail est surtout pratiqué par quelques professions intellectuelles et/ou caractérisées par une forte autonomie (Schampheleire et Martinez, 2006). Si la moyenne nationale des télétravailleurs à domicile est de $8 \%$ selon l'ENTD, les professions dans lesquelles les télétravailleurs sont les plus représentés sont les chefs d'entreprise (41\%), les professions libérales (32\%), et enfin les cadres (du privé et du public) mais aussi les agriculteurs/exploitants, où un cinquième environ des personnes déclarent faire du télétravail. L'enquête bretonne confirme ces chiffres : parmi les PME qui déclarent pratiquer le télétravail, $73 \%$ déclarent que cela concerne des cadres de l'entreprise, $25 \%$ des professions intermédiaires, $25 \%$ des employés et seulement $13 \%$ des ouvriers.

Un résultat plus original est que, selon l'ENTD, les habitants de Paris sont très fortement surreprésentés dans la population des télétravailleurs français. En effet la part des télétravailleurs à domicile varie assez peu en fonction de la taille du lieu de résidence ou de son caractère urbain, périurbain ou rural, sauf à Paris (mais pas dans le reste de l'île de France), où cette part est de $18 \%$ soit plus du double de la moyenne nationale. La concentration de professions supérieures mais aussi les difficultés de déplacements sont probablement les deux principales explications. En effet la part des télétravailleurs augmente avec le temps de trajet domiciletravail, et atteint $13 \%$ lorsque ce trajet dépasse 60 minutes. Le même effet ressort de l'enquête bretonne: le télétravail au sens large ne croît pas linéairement avec la distance mais la 
pratique est plus fréquente dès qu'on passe le seul d'un trajet domicile-travail de plus de 30 minutes. En revanche, on ne voit pas apparaître d'effet agglomération au niveau de la région Bretagne : la pratique du télétravail n'est pas liée à la localisation géographique (urbaine, périurbaine ou rurale) de l'entreprise, probablement car les difficultés de circulation restent globalement modérées dans cette Région.

\subsection{Un potentiel de développement très limité}

Les résultats de nos travaux ne vont pas dans le sens d'une forte augmentation de la pratique du télétravail, contrairement aux prévisions particulièrement optimistes rappelées en introduction. En tout cas, l'exemple breton suggère que le potentiel de développement dans les petites et moyennes entreprises localisées en province est particulièrement faible. Tout d'abord, parmi les entreprises qui n'ont pas mis en place le télétravail, seulement 2,4\% déclarent projeter de le faire. Nous pouvons cependant supposer que, la pratique du télétravail étant peu répendue dans les PME, celles qui ne l'ont pas mis en place ne sont pas encore en mesure de percevoir les futurs bénéfices de cette pratique.

En revanche, parmi celles qui l'ont déjà mis en place, 76,9 \% déclarent ne pas vouloir développer le télétravail, et 3,7\% déclarent même vouloir le réduire! Seuls $15 \%$ des entreprises disent vouloir l'étendre : 11,4\% souhaitent qu'il concerne plus de salariés, et 4,5\% souhaitent augmenter le temps de travail à distance mais sans augmenter le nombre de télétravailleurs. La volonté d'étendre le télétravail ne dépend ni du nombre de salariés concernés dans l'entreprise, ni de la fréquence à laquelle les salariés travaillent en dehors des locaux de l'entreprise. La mise en place de cette pratique ne semble donc pas répondre à une dynamique, qui laisserait augurer un développement futur.

\section{Des freins du côté des entreprises et des salariés}

Les travaux qui s'intéressent aux facteurs favorables et aux freins à la mise en place du télétravail sont nombreux (De Beer, 2006). Si les résultats sont contrastés, la tonalité générale est celle du constat de réticences assez fortes et d'une motivation très limitée à la fois des entreprises et des salariés expliquant d'ailleurs la faible diffusion du télétravail alors que les technologies sont disponibles depuis plusieurs années. Les enquêtes menées en Bretagne confirment ces constats, et en explicitent certaines des raisons.

\subsection{La nécessité de réorganiser le travail}


Plusieurs auteurs soulignent que la diffusion du télétravail à domicile ne peut se faire sans l'implémentation de changements organisationnels conséquents (Illegems et al., 2001), voire d'une nouvelle culture d'entreprise (Storhaye et Bouvard, 2013). Selon Taskin (2006), le véritable enjeu du télétravail est en fait la capacité à gérer la déspatialisation, c'est-à-dire la distance non seulement physique (géographique) du travailleur, mais aussi et surtout psychosociologique, liée à l'éloignement d'avec son environnement de travail au sens large (collègues, espaces communs, échanges informels et formels, etc.). Or cette distance nécessite de repenser l'organisation du travail.

Les entreprises bretonnes perçoivent bien la difficulté de la gestion à distance des salariés, et les changements qu'elle implique. La nécessaire présence du salarié dans les locaux de l'entreprise est en effet la raison la plus souvent invoquée par les entreprises qui ne pratiquent pas le télétravail (88\% d'entre elles, et plus de 58\% des entreprises voient dans la nécessité d'une réorganisation du travail un inconvénient très important ou assez important à la mise en place de cette pratique ${ }^{10}$.

Les entreprises qui estiment que la réorganisation du travail n'est pas un inconvénient important à la mise en place du télétravail sont surreprésentées parmi celles qui l'ont effectivement mis en place, alors que celles qui estiment que c'est un frein important ou très important sont surreprésentées au sein des non-pratiquantes. Pour une partie des entreprises, le télétravail impliquerait des contraintes organisationnelles qui semblent rédhibitoires.

Pourtant, seule une part très faible des entreprises qui pratiquent le télétravail $(7,8 \%)$ déclarent que cela a impliqué une réorganisation du travail. Une première justification à ce résultat est qu'une part importante des entreprises qui ont mis en place le télétravail accueillent des travailleurs nomades (commerciaux, livreurs, ...), pour lesquels la possibilité de travailler en dehors des locaux constitue est déjà une caractéristique de leur organisation. Elles auraient donc déjà mis en place des formes d'organisation adaptées aux pratiques multiples du travail occasionnel ou régulier à distance.

\subsection{Des changements nécessaires dans les pratiques de management}

La littérature montre que l'accent mis par les entreprises sur la nécessaire présence physique des salariés n'est pas qu'une question de nature des tâches à accomplir, mais tient aussi aux difficultés de conduite du management à distance (Taskin, 2006, Illegems et Verbeke, 2004),

\footnotetext{
${ }^{10}$ Les questions portant sur les avantages et les inconvénients de la mise en place du travail à distance ont été posées à l'ensemble des entreprises interrogées, afin de mesurer les effets réellement perçus et les effets potentiels. Une part importante des entreprises qui ne pratiquent pas le travail à distance n'ont pas répondu à ces questions. Les statistiques présentées ont été calculées sur les entreprises ayant répondu à ces questions (669 concernant les avantages et 696 concernant les inconvénients).
} 
ainsi qu'à des facteurs individuels, dont l'isolement social et la perte de l'esprit d'équipe (Thomsin et Tremblay, 2006). Du point de vue de l'employeur, le télétravail présente le désavantage de soustraire la personne au contrôle physique de son supérieur (Felstead et al, 2003). L'instauration du télétravail peut alors s'accompagner d'une nouvelle forme de contrôle du salarié, qui peut passer par la mise en place de moyens technologiques (Fairweather, 1999). Elle peut aussi s’appuyer sur une plus grande autonomie.

Une des raisons les plus souvent invoquées par les entreprises bretonnes pour justifier le fait de ne pas pratiquer le télétravail est que cela n'est pas compatible avec leur mode de management (raison jugée assez ou très importante par 77\% d'entre elles). Seule la moitié des entreprises $(52 \%)$ font directement référence au problème du contrôle du travail réalisé. La limitation des interactions entre le salarié et ses collègues est aussi une limite très souvent avancée (66\% des firmes pensent que c'est un inconvénient important ou très important), autant par les entreprises qui pratiquent le télétravail que par celles qui ne le pratiquent pas.

$\mathrm{Si}$, comme nous l'avons déjà souligné dans la partie précédente, seule une faible part des entreprises déclarent que la mise en place du télétravail s'est accompagnée d'une réorganisation du travail, pour la grande majorité d'entre elles (16 sur 19) cette réorganisation s'est faite par l'intermédiaire d'une plus grande autonomie du travailleur. La mise en place d'un mode de supervision à distance reste très peu pratiqué (par seulement 3 entreprises sur 19). Malgré tout certains outils technologiques peuvent être utilisés afin de combler la distance qui sépare le télétravailleur de son lieu de travail. Si 43,2\% des entreprises exigent que leurs télétravailleurs soient joignables en permanence par téléphone lorsqu'ils travaillent à distance, 27,6\% qu'ils répondent dans des délais courts à leurs mails et 11,9\% qu'ils soient connectés en permanence au réseau de l'entreprise, presque la moitié des entreprises concernées $(49,8 \%)$ ne formulent aucune exigence. Dans les entreprises enquêtées, la gestion du travail à distance passe donc souvent par une plus grande autonomie du travailleur et ne repose pas nécessairement sur des règles très strictes.

Enfin, la mise en place du télétravail à domicile, qui ne peut concerner toutes les catégories de salariés, peut par ailleurs créer des tensions, le télétravail étant considéré comme une forme de privilège (Le Gléonnec, 2001). Cet inconvénient est évoqué par $44 \%$ des entreprises que nous avons enquêtées.

\subsection{Un gain versus une perte d'efficacité des salariés}


Des gains de productivité de la part des télétravailleurs sont parfois attendus, notamment du fait d'une réduction de l'absentéisme liée aux facilités d'organisation de la vie privée (Kitou et Horvath, 2008). A l'inverse, des travaux empiriques mettent en évidence un effet différencié du télétravail sur la productivité, qui dépend en particulier de la qualité des interactions avec les managers et avec les membres de la famille (Neufeld et Fang, 2005).

L'enquête régionale réalisée auprès les PME montre que la perception des liens entre télétravail et efficacité des salariés constitue un facteur influent. Les entreprises qui ne pratiquent pas le télétravail sont ainsi beaucoup plus nombreuses à penser que le télétravail n'augmente pas l'efficacité des salariés: parmi les entreprises qui ne pratiquent pas le télétravail, $80 \%$ estiment que l'augmentation de l'efficacité des salariés liée au télétravail est un avantage peu ou pas important, contre seulement $43 \%$ parmi les entreprises qui l'ont mis en place. Pour certaines, la mise en place du télétravail conduit ou conduirait même à une baisse de l'efficacité des salariés : $44 \%$ des entreprises qui ne pratiquent pas le télétravail pensent que la réduction de l'efficacité des travailleurs est un inconvénient important ou très important, contre $26 \%$ des entreprises qui ont mis en place cette pratique. L'opinion des entreprises concernant l'effet du télétravail sur l'efficacité des télétravailleurs est un des facteurs qui différencie non seulement les entreprises qui pratiquent le télétravail et celles qui ne pratiquent pas, mais aussi celles qui déclarent vouloir l'étendre, qui y voient des gains d'efficacité, et celles qui ne le veulent pas, voire souhaitent le réduire, et qui en général estiment que cela implique une perte d'efficacité des travailleurs.

Du côté des salariés, le lien entre travail à domicile et gains d'efficacité n'est pas non plus unanime. En effet, seulement $22 \%$ des actifs qui télétravaillent considèrent qu'ils sont plus efficaces à leur domicile que sur leur lieu de travail...

L'appréciation du lien entre efficacité et télétravail est donc ambiguë, même parmi les entreprises qui ont mis en place cette pratique et les salariés qui y ont recours...Des enquêtes plus qualitatives seraient nécessaires pour mieux en comprendre les raisons, qui ne tiennent probablement pas seulement aux aspects professionnels mais également aux caractéristiques de la vie privée des télétravailleurs (présence d'enfants, type d'emploi du conjoint, etc.).

\subsection{L'importance de la dimension technologique}

La généralisation de l'utilisation des TIC au sein des ménages et des entreprises, a souvent été avancée comme un levier attendu du développement du télétravail (Bayrak, 2012; Richardson 
et Benbunan-Fich, 2011). Les enquêtes réalisées en Bretagne suggèrent que les freins technologiques liés au télétravail concernent plus les entreprises que les salariés. Parmi ces derniers, $2 \%$ seulement mettent en effet en avant un manque d'outils technologiques comme frein au travail à domicile. De fait, les télétravailleurs ont principalement besoin pour leur activité à domicile d'équipements devenus désormais relativement standards : un ordinateur fixe ou portable (pour 94\% d'entre eux), une connexion à Internet (pour 81,4\%), et un téléphone fixe ou portable $(53 \%)$.

Du côté des entreprises, si la présence d'outils technologiques adaptés en interne et la maîtrise de ces outils par les salariés sont rarement avancés comme des freins par celles qui ne pratiquent pas le télétravail, la majorité des entreprises sont conscientes que la mise en place du télétravail nécessite des investissements technologiques spécifiques. 51\% des entreprises interrogées jugent que la nécessité de disposer d'équipements technologiques spécifiques est un inconvénient assez ou très important à la mise en place du télétrévail. Ce sentiment est, de surcroît, partagé aussi bien par les entreprises qui pratiquent le télétravail (et dont la moitié a dû réaliser des investissements technologiques spécifiques dans le cadre de sa mise en place), que par celles qui ne le pratiquent pas. Le frein technologique à la mise en place du télétravail est donc fort pour les entreprises, mais pas rédhibitoire.

\subsection{Une pratique peu plébiscitée, autant pas les salariés que par les entreprises}

Selon Bailey et Kurlet, 2002, les motivations des salariés pour le télétravail ne sont pas claires. Le principal argument avancé par les actifs bretons concernant les raisons pour lesquelles ils ne travaillent pas à distance est que leur métier ne le permet pas $(93 \%$ de l'échantillon). Les autres freins possibles (opposition de l'employeur, absence des outils de communication adéquats au domicile, volonté de séparer la vie privée et la vie professionnelle) sont très peu cités dans notre enquête (par 2 à $4 \%$ des actifs). Du côté des entreprises, la réticence des managers, fondée principalement sur des préoccupations de coût d'investissement, d'organisation et de contrôle du travail et renforcé par un faible besoin perçu, limite le développement de la pratique.

Par ailleurs un parallèle entre, d'une part le principal frein mis en avant par les entreprises (c'est-à-dire la nécessaire présence des salariés sur le site), et d'autre part la proportion importante, dans nos enquêtes, de non réponses aux questions portant sur les inconvénients et les avantages potentiels du télétravail de la part des entreprises non pratiquantes, laisse supposer que pour une part non négligeables des PME interrogées le télétravail n'est en réalité tout simplement pas envisagée. Autrement dit, une part importante des entreprises et des 
salariés interrogés partent du postulat que le télétravail ne leur est pas accessible, sans chercher précisément à en évaluer les potentialités.

\section{Des gains sociaux et environnementaux incertains}

Les espoirs placés dans le télétravail à domicile ou en télécentres sont à peu près inchangés depuis quarante ans (De Beer, 2006). Ils sont principalement de deux ordres : premièrement une meilleure conciliation entre vie privée et vie professionnelle, et deuxièmement des gains environnementaux, qui passent notamment par une diminution du volume des déplacements domicile-travail.

\subsection{Un impact mitigé sur l'articulation entre vie privée et vie professionnelle}

Le télétravail est envisagé pour réduire les tensions croissantes entre vie privée et vie professionnelle (Di Martino et Wirth, 1990 ; Metzger et Cléach, 2004 ; Tremblay, 2003). Ces tensions sont en particulier fortes chez les femmes (Fagnani et al., 2004), les cadres (Guillaume et Pocchic, 2009), les personnes soumises à de longs trajets domicile-travail (Helminen et Ristimaki, 2007 ; Mokhtarian et al., 2004 ; Peters et al, 2004 ; Setow, 2014), ou encore à des horaires de travail longs ou décalés (Mc Innes, 2005).

Les études empiriques apportent toutefois des réponses ambiguës et pointent notamment le danger d'envahissement de la vie privée (Maruyama, Hopkinson, James, 2009 ; Tremblay et al., 2006). L'enquête réalisée auprès des individus bretons montre d'ailleurs que seulement $12 \%$ des individus qui télétravaillent à leur domicile (au sens strict) déclarent que cela leur permet de mieux équilibrer leur vie privée et leur vie professionnelle.

\subsection{Des gains environnementaux discutables}

Les gains environnementaux, qui concentrent des attentes sociétales croissantes dans le cadre de la lutte internationale contre le changement climatique, sont tout autant controversés dans la littérature. Premièrement, le télétravail à domicile ou en télécentres permet de réduire la consommation énergétique des entreprises grâce à une utilisation optimale des locaux (Matthews et Williams, 2005). Il faut toutefois tenir compte du surcroît de consommation occasionné dans les logements des salariés qui vont être amenés à travailler chez eux un ou plusieurs jours par semaine. Les estimations des gains énergétiques faits par Matthews et Williams pour les USA et le Japon montrent des économies très modestes, inférieurs à $0,4 \%$ 
dans la situation actuelle et atteignant au plus $1 \%$ sous l'hypothèse que les salariés qui peuvent télétravailler restent chez eux 4 jours par semaine.

Deuxièmement, les gains escomptés en matière de déplacements domicile-travail semblent tout aussi fragiles. Ces gains reposent sur trois hypothèses: la première est que les télétravailleurs sont surtout des gens qui utilisent leur voiture pour aller sur leur lieu d'emploi, sinon le gain n'est pas annulé mais plus ou moins fortement amoindri, et/ou que le fait de pouvoir télétravailler va encourager certains actifs à abandonner l'automobile, en particulier dans les grandes villes du fait de la qualité et de la densité de l'offre de transports en commun. Les données dont nous disposons ne permettent pas de vérifier cette hypothèse. L'ENTD révèle seulement que les télétravailleurs (occasionnels ou réguliers) sont un peu plus de $71 \%$ à aller travailler en voiture, contre $76 \%$ pour les non télétravailleurs. Cette différence ne nous permet pas de conclure, car elle probablement expliquée principalement par le fait que dans les grandes villes, et notamment en Île de France, la part des transports en commun augmente avec la durée domicile-travail (Schwanen et Dijst, 2002, Korsu et Massot, 2006), or nous avons vu que cette durée est plus importante pour les télétravailleurs.

La seconde hypothèse est que les personnes qui télétravaillent à domicile ne vont pas avoir tendance, à court ou moyen terme, à augmenter leurs déplacements pour motifs privés (achats, loisirs, accompagnement) en profitant des gains de temps offerts par l'évitement de certains trajets et de leur présence à leur domicile: en effet les lieux de vie (école, commerces, banque, etc.) demeurent fortement polarisés par le lieu de résidence, bien plus que par le lieu de travail (Boulahbal, 2001). Or plusieurs travaux confirment qu'une partie des télétravailleurs tendent à augmenter leurs déplacements privés (Arnfalk, 2002; Moos et al., 2006), tandis qu'une autre partie ne change rien à sa mobilité ou augmente ses déplacements professionnels (Walls et Saforiva, 2004). Au final, on constate quand même à court ou moyen termes une baisse du volume total des kilomètres parcourus après le passage au télétravail dans la majorité des cas (Perez et al., 2004 ; Walls et Saforiva, 2004). Toutefois cette baisse est souvent très limitée : elle est par exemple estimée à moins de $1 \%$ des kilomètres parcourus en voiture aux USA (Choo, Mokhtarian et Salomon, 2005). Dans l'enquête réalisée en Bretagne, si seulement 2,5\% des télétravailleurs à domicile avancent comme motivation la volonté de réduire la fréquence des trajets domicile-travail, on observe que $84 \%$ d'entre eux estiment se déplacer moins les jours où ils travaillent à leur domicile contre $8,5 \%$ qui déclarent se déplacer autant et 7,5\% se déplacer davantage, ce qui confirme les résultats des autres études. Toutefois il faut rester prudent dans la mesure où cette enquête ne permet pas 
une estimation véritablement précise de la mobilité des télétravailleurs avant et après leur passage au télétravail.

La troisième hypothèse est que les localisations (des ménages voire des entreprises) ne vont pas à long terme évoluer avec la diffusion du télétravail. Or un scénario envisageable est que le télétravail implique une déconcentration des ménages sur le long terme (Janelle, 1986; Nilles, 1991), et donc augmente la distance parcourue pour les trajets hors travail et les trajets domicile-travail (les jours où la personne se rend sur son lieu de travail), tout en réduisant la congestion aux heures de pointe (Van Horn et Storen, 2000). D'ores et déjà, plusieurs études constatent que la distance moyenne domicile-travail de ceux qui pratiquent le télétravail est plus élevée que celle des autres travailleurs (Mokhtarian et al., 2004; Peters et al., 2004, Helminen et Ristimaki, 2007), ce que confirme aussi l'ENTD : la distance moyenne domiciletravail des actifs français pratiquant régulièrement ou occasionnellement le télétravail atteint 19 kilomètres en 2008, contre un peu moins de 15 kilomètres pour les non télétravailleurs. Toutefois il est difficile de dire avec ces seules données si la pratique du télétravail est liée à des distances (et des temps) de navettage élevées, ou si le fait de pouvoir télétravailler encourage à s'éloigner de son lieu d'emploi.

\section{Conclusion}

Nous nous sommes interrogés sur les raisons du décalage important entre les fortes attentes dont fait l'objet le télétravail et la faiblesse de sa pratique. Les trois sources statistiques utilisées confirment tout d'abord non seulement le faible niveau de développement actuel du télétravail au sens où nous l'avons défini, mais aussi l'absence d'une réelle dynamique. L'enquête régionale menée en Bretagne montre notamment qu'un faible nombre d'entreprises, parmi celles qui ne pratiquent pas le télétravail, souhaite l'adopter. Pire encore : parmi les entreprises qui l'ont mis en place, peu souhaitent poursuivre l'expérience....

Cette situation frappe par sa récurrence. Elle ne date pas en effet d'aujourd'hui, comme si le télétravail était structurellement prisonnier d'un équilibre de bas niveau. En conséquence, l'argument qui consiste à peindre et à repeindre sans cesse le télétravail aux couleurs de son improbable futur peine à convaincre. Nous avons en effet montré que l'équilibre de bas niveau s'explique par l'existence de verrous solides tant du côté des entreprises que des salariés. Du côté des entreprises, les coûts de la mise en place du télétravail sont vivement perçus (dans notre enquête, il s'agit principalement des coûts de changement organisationnels et les coûts technologiques), tandis qu'elles ont des doutes sur les gains d'efficacité apportés. 
Du côté des salariés, le télétravail est aujourd'hui limité à des catégories spécifiques de travailleurs (celles dont l'autonomie déjà acquise n'implique pas véritablement de changement organisationnel et managérial), et n'est pas nécessairement perçu comme un facteur de meilleur équilibre entre vie privée et professionnelle ou source d'une efficacité accrue. Ce double manque d'attrait pour le télétravail le pénalise d'autant plus que les gains environnementaux n'apparaissent pas non plus très évidents, dès lors qu'on les examine rigoureusement, c'est à dire en considérant l'ensemble des mobilités des individus.

Pour dépasser ces blocages, soulignons tout d'abord que le potentiel de développement du télétravail nous semble surtout concerner les grandes métropoles, comme le montre d'ailleurs la sur-représentation actuelle des habitants de Paris dans la population des télétravailleurs en France. La raison principale est que le temps de transport y est beaucoup plus élevé qu'ailleurs, pour tous les types de déplacement ( $82 \mathrm{mn}$ en moyenne par jour en Ile de France contre $64 \mathrm{mn}$ en province) comme pour les déplacements domicile-travail (plus d'une demi heure en moyenne par jour contre $17 \mathrm{mn}$ en province). Plus de $22 \%$ des habitants de l'Ile de France consacrent plus de $2 \mathrm{~h}$ à leurs déplacements par jour contre 12\% en France.

Le développement du télétravail informel, basé sur des arrangements entre employeur et salarié, est une première réponse. Nos enquêtes montrent qu'il est déjà développé, bien qu'il soit difficile de le mesurer. Son succès tient à son absence de formalisation. Cette dernière élève en effet les coûts d'entrée dans le télétravail, en particulier du point de vue des entreprises: adoption de nouvelles procédures organisationnelles, apport de garanties juridiques, aménagement de nouveaux postes de travail... Or, compte-tenu du faible intérêt intrinsèque qu'elles lui accordent, ces coûts sont dissuasifs pour les entreprises, au moins les PME.

Lorsque les pratiques informelles de télétravail sont tacitement acceptées, les salariés y ont aussi intérêt. Ils ne s'engagent en effet pas formellement dans un nouveau statut qui peut être risqué, surtout en période de crise économique, tout en pouvant à l'occasion télétravailler. Naturellement, cette possibilité est limitée et n'est pas ouverte à tous les salariés. Mais il serait intéressant de pouvoir en mesurer le développement. Cela montrerait que le télétravail informel est pratiqué comme une variable d'ajustement dans certaines situations contraintes, personnelles ou collectives, par exemple dans les agglomérations fortement congestionnées.

Le développement du télétravail formel supposerait d'autres conditions qui feraient basculer les dilemmes coût-avantage que nous avons décrits. Cela serait le cas si les pouvoirs publics 
décidaient une politique plus forte d'internalisation par les acteurs privés des coûts sociaux engendrés par la congestion (taxes carburants, taxes transport, péages urbains...), les contraignant à réviser leurs choix actuels. Une brutale hausse des carburants aurait un effet analogue. Le développement de formes organisées du télétravail apparaîtrait alors comme une alternative nécessaire, en particulier pour ceux qui subissent déjà mais subiraient encore plus durement de longs et coûteux temps de déplacement. Sans un choc exogène de cet ordre, le télétravail restera vraisemblablement ce qu'il est aujourd'hui.

\section{Références}

Aguiléra A, Delaplace M, Perrin J. (2013) Les espaces de travail temporaires dans les gares TGV: Une innovation de service pour la conquête du marché métropolitain des professionnels mobiles. In Colloque International du LabeX Futurs Urbains: Métropoles et aménagements métropolitains: quelles dynamiques d'innovations?

Arnfalk P (2002) Virtual Mobility et Pollution Prevention-The Emerging Role of ICT Based Communication in Organisations et its Impact on Travel (Vol. 2002, No. 1). Lund University.

Bailey DE, Kurlet N B (2002) A review of telework research: Findings, new directions, et lessons for the study of modern work. Journal of organizational behavior, 23(4): 383-400.

Bathelt H, Schuldt N (2008) Between luminaries et meat grinders: International trade fairs as temporary clusters. Regional Studies 42: 853-868.

Bayrak, T. (2012) IT support for Telecommuting Workforce. Telematics et Informatics, 29: 286-293.

Boboc A, Dhaleine L, Mallard A (2007. Travailler, se déplacer et communiquer: premiers resultats d'enquête. Réseaux 1 : 133-158.

Boboc A, Dhaleine L (2008) Faire du privé au bureau, une question de genre ? Réseaux 6 : 393-416.

Boulahbal M (2001) Effet polarisant du lieu de travail sur le territoire de la vie quotidienne des actifs. Recherche, Transports, Sécurité, 73 : 43-63.

Choo S, Mokhtarian PL, Salomon I (2005) Does telecommuting reduce vehicle-miles traveled? An aggregate time series analysis for the US. Transportation 32(1): 37-64.

Crague G (2003). Des lieux de travail de plus en plus variables et temporaires. Economie et statistique, 369(1) : 191-212.

De Beer A (2006) Le télétravail en perspective. Futuribles, 317 : 59-78.

Devillers M. (2003) Le télétravail: dossier de synthèse documentaire.

Di Martino V, Wirth L (1990) Telework: A new way of working and living. Interntional Labour Review, 129 (5): 529-554.

Fagnani J, Giovannini D, Højgaard L, Clarke H (2004) Fathers et mothers: Dilemmas of the work-life balance: a comparative study in four European countries, Springer Science and Business Media, 21.

Fairweather N B (1999) Surveillance in employment: The case of teleworking. Journal of Business Ethics, 22(1): 39-49. 
Farrah BJ, Dagen CD (1993) Telecommuting Policies That Work, HR Magazine 38: 64-71

Felstead A, Jewson N, Walters S (2003). Managerial control of employees working at home. British Journal of Industrial Relations, 41(2): 241-264.

Guillaume, C., Pochic, S. (2009). What would you sacrifice? Access to top management et the work-life balance. Gender, Work \& Organization16(1), 14-36.

Helminen V, Ristimäki M (2007). Relationships between commuting distance, frequency and telework in Finland. Journal of Transport Geography, 15(5): 331-342.

Huws U (1984) The New Homeworkers : New Technology et the Changing Location of White Collar Work, Low Pay Unit, London:UK.

INSEE (2009) E-administration, télétravail, logiciels libres : quels usages de l'internet dans les entreprises? INSEE Première.

Illegems V, Verbeke A (2004) Telework: what does it mean for management? Long Range Planning 37(4): 319-334.

Illegems V, Verbeke A, S'Jegers R (2001) The organizational context of teleworking implementation. Technological Forecasting and Social Change, 68(3): 275-291.

Janelle D G, 1986, "Metropolitan expansion et the communications transportation tradeoff", in The Geography of Urban Transportation Ed. S Hanson (Guilford Press, New York), 357-385.

Kitou E, Horvath A (2008) External air pollution costs of telework. The International Journal of Life Cycle Assessment, 13(2): 155-165.

Korsu E, Massot MH (2006) Rapprocher les ménages de leurs lieux de travail: les enjeux pour la régulation de l'usage de la voiture en Île-de-France. Les Cahiers scientifiques du transport, $50: 61-90$.

Gléonnec M. (2001) Le changement organisationnel à l'épreuve du lien social dans ses modalités culturelles: l'appropriation du télétravail dans l'entreprise, Thèse de doctorat, Bordeaux 3.

MacInnes J (2005) Work-life balance and the demand for reduction in working hours: Evidence from the British Social Attitudes Survey 2002. British Journal of Industrial Relations 43(2): 273-295.

Maruyama T, Hopkinson PG, James PW (2009) A multivariate analysis of work-life balance outcomes from a large scale telework programme. New Technology, Work and Employment, 24(1): 76-88.

Matthews HS, Williams E (2005) Telework adoption et energy use in building and transport sectors in the United States et Japan. Journal of infrastructure systems, 11(1) : 21-30.

Metzger JL, Cléach O (2004) Le télétravail des cadres: entre suractivité et apprentissage de nouvelles temporalités. Sociologie du travail, 46(4) : 433-450.

Mokhtarian PL, Collantes GO, Gertz C (2004) Telecommuting, residential location and commute distance travelled: Evidence from State of California employees. Environment and Planning A 36(10): 1877 - 1897.

Moos M, Etrey J, Johnson LC (2006) The sustainability of telework: an ecologicalfootprinting approach. Sustainability: Science Practice and Policy 2(1): 3-14. 
Morel-A-Lhuissier P (2007) Du télétravail au travail mobile: Un enjeu de modernisation de l'économie française. Rapport au premier ministre, La Documentation française.

Moriset B (2004) Télétravail, travail nomade: le territoire et les territorialités face aux nouvelles flexibilités spatio-temporelles du travail et de la production. Cybergeo: European Journal of Geography Online since 06 February 2004, connection on 23 June 2015. URL: http://cybergeo.revues.org/3815 ; DOI : 10.4000/cybergeo.3815.

Neirotti P, Paolucci E, Raguseo E (2013) Mapping the antecedents of telework diffusion: firm level evidence from Italy. New Technology, Work et Employment 28(1): 16-36.

Nilles J M (1991) Telecommuting et urban sprawl: Mitigator or inciter?, Transportation 18: 411-432

Peters P, Tijdens KG, Wetzels C (2004) Employees' Opportunities, Preferences, et Practices in Telecommuting Adoption. Information \& Management 41: 469-482.

Pérez MP, Sánchez AM, de Luis Carnicer MP, Jiménez MJV (2004) The environmental impacts of teleworking: A model of urban analysis et a case study. Management of Environmental Quality: An International Journal 15(6): 656-671.

Rallet A, Torre A (2009) Temporary geographical proximity for business et work coordination: When, how et where? SPACES online (2009-02), Toronto, Heidelberg: www.spaces-online.com.

Richardson K, Benbunan-Fich R (2011) Examining the antecedents of work connectivity behavior during non-work time. Information and Organisation, 21: 142-160.

Setow E (2014) Til work do us part: the social fallacy of long-distance commuting. Urban Studies 51(3) : 526-543.

Schampheleire JD, Martinez E (2006). Régulation du télétravail et dialogue social. Le cas de la Belgique. Revue Interventions économiques. Papers in Political Economy, 34, mis en ligne le 01 juillet 2006, consulté le 23 juin 2015. URL: http://interventionseconomiques.revues.org/659

Schwanen T, Dijst M (2002) Travel-time ratios for visits to the workplace: the relationship between commuting time et work duration. Transportation Research A, 36 : 573-592.

Storhaye $\mathrm{P}$, Bouvard $\mathrm{P}$ (2013) Le travail à distance: Télétravail et nomadisme, leviers de transformation des entreprises. Dunod.

Taskin L. (2006) Télétravail: Les enjeux de la déspatialisation pour le Management Humain. Revue Interventions économiques. Papers in Political Economy, 34.

Taskin L, Vendramin P (2004) Le télétravail, une vague silencieuse : Enjeux socioéconomiques d'une nouvelle flexibilité. Louvain-la-Neuve : Presses Universitaires de Louvain.

Torre A (2008) On the role played by temporary geographical proximity in knowledge transmission. Regional Studies 42: 869-889.

Thomsin L, Tremblay DG (2008) Exploring the diversity of mobile working: a detailed examination on the sequences of workplaces et job satisfaction. Journal of eWorking, 2(1).

Tremblay DG, Chevrier C, Loreto MD (2006) Le télétravail à domicile: Meilleure conciliation emploi-famille ou source d'envahissement de la vie privée? Revue Interventions économiques. Papers in Political Economy, 34. 
Van Horn CE, Storen D (2000) Telework: Coming of age? Evaluating the potential benefits of telework. In Telework: The new workplace of the 21st Century symposium, New Orleans.

Walls MA, Safirova E (2004) A review of the literature on telecommuting et its implications for vehicle travel et emissions. Resources for the Future. 